\title{
IN GOD WE TRUST: SOBRE LAS RAÍCES CRISTIANAS DEL CONCEPTO DE LIBERTAD RELIGIOSA EN LOS ESTADOS UNIDOS DE AMÉRICA
}

\author{
Carlos Piccone Camere, OFMCap. \\ Universidad de Londres \\ c.picconecamere@postgrad.sas.ac.uk
}

Fecha de recepción: agosto de 2019 Fecha de aceptación: diciembre de 2019

ReSUMEN: De manera sucinta y panorámica, el presente artículo presenta el concepto de libertad religiosa en los Estados Unidos de América, desde una perspectiva histórica. Se trata de un valor esencial que los estadounidenses recibieron como un legado del espíritu cristiano de los primeros colonos y la notable influencia de las corrientes inglesas de pensamiento durante el siglo XVII. Si bien la libertad fue el valor que los primeros colonos adoptaron como una piedra angular capaz de sostener sus textos legislativos y sus relaciones interpersonales, ha predominado en el imaginario norteamericano la construcción historiográfica de los Padres Peregrinos como la personificación de dicho valor. La vigencia del tema se ve confirmada ante el auge actual de ciertos tipos de fundamentalismos religiosos. En este contexto, se enfatiza el sano equilibrio que debe tener el Estado para cumplir con un doble deber:

Carlos Piccone Camere, OFMCap. es licenciado en Historia de la Iglesia por la Universidad Gregoriana de Roma. Se ha desempeñado como docente en la Universidad San José (Lima) y en la Escuela Superior de Estudios Franciscanos (Madrid). Actualmente realiza un doctorado en Estudios Latinoamericanos en la Universidad de Londres. 
por un lado, la defensa de la libertad religiosa y, por el otro, el de no cejar en el empeño de erradicar cualquier tipo de violencia que pretenda justificar sus aberraciones utilizando el nombre de Dios.

Palabras Clave: Libertad religiosa, Historia de la Iglesia, Estados Unidos de América.

\section{IN GOD WE TRUST: ON THE CHRISTIAN ROOTS OF THE CONCEPT OF RELIGIOUS FREEDOM IN THE UNITED STATES OF AMERICA}

AвSTRACT: Through a panoramic overview, this article presents the concept of religious freedom in the United States of America from a historical perspective. Americans received freedom as an essential value inherited from the Christian spirit of the first settlers and the remarkable influence of the currents of thought in Seventeenth-Century England. Although freedom was the value that early American settlers adopted as a cornerstone, in order to sustain their legislative texts and interpersonal relationship, the historiographical construction of the Pilgrim Fathers as the personification of liberty has predominated in the American social imaginary. The topic has gained renewed importance because of the current rise of certain forms of religious fundamentalisms. In this context, it is emphasized the fair balance that the State must carry out in order to fulfill a double duty: the defense of religious freedom, as well as the eradication of any kind of violence that would try to justify its own aberrations by misusing the name of God. KeYwords: Religious freedom, Church History, The United States of America. 
IN GOD WE TRUST: SOBRE LAS RAÍCES CRISTIANAS DEL CONCEPTO DE LIBERTAD RELIGIOSA EN LOS ESTADOS UNIDOS DE AMÉRICA

If we ever forget that we are one nation under God, then we will be a nation gone under. ${ }^{1}$

Ronald Reagan

\section{Antecedentes}

7 n la denominada Carta sobre la Tolerancia, John Locke postuló algunas de las bases ideológicas de su teoría política sobre la naturaleza de la sociedad y del gobierno². Locke defendió el imperio de la conciencia del individuo en el medio social y en el sistema político. En su opinión, el hombre está dotado de suficiente libertad como para ser capaz de elegir la profesión de su fe dentro de una determinada religión (Locke, [1689] 2013, p. 78). Como consecuencia de lo anterior, el Estado debía renunciar a sus pretensiones de imponer a los ciudadanos una legislación en materia religiosa. Estos postulados contrastaban con la antropología pesimista de Thomas Hobbes quien, décadas atrás, había publicado su célebre obra Leviatán o la materia, forma y poder de una república eclesiástica $y$ civil (1651).

Por otra parte, en la exposición de sus ideas, John Locke hacía alusión a la rebelión del movimiento puritano acaecido en su tierra natal. Según el pensador inglés, dicho evento habría sido una de las principales causas de la revolución de Oliver Cromwell y, eventualmente, permitiría un reconocimiento de facto de las restantes religiones institucionalizadas, a las que el padre del Liberalismo Clásico englobaba bajo el nombre de "sectas

\footnotetext{
1 "Si alguna vez olvidamos que somos una nación bajo Dios, entonces seremos una nación que ha caído bajo" (Reagan, 1986, p. 1168).

2 John Locke (1632-1704), uno de los más influyentes filósofos del empirismo inglés y precursor del racionalismo (Forster, 2005, p. 13). También destacaron en Inglaterra reformadores de la talla de Roger Williams y John Milton, de cuyos pensamientos se hicieron eco Sebastián Castellion y Hugo Grocio, entre otros (Glaser, 2014, p. I).
} 
protestantes" (Marshall, 2006, pp. 94-95). En efecto, cierta historiografía postula que aquella rebelión de los puritanos ingleses habría allanado el camino hacia la instauración de una especie de semitolerancia religiosa; lo cual habría jugado a favor del pluralismo religioso dentro del Estado (Armstrong \& Tennenhouse, 1992, p. 51).

\section{La Libertad Religiosa en los Estados Unidos de América}

$\mathrm{P}$ ues bien, no es casualidad que uno de los hilos conductores más sólidos en los inicios de la historia de los Estados Unidos de América hubiese sido, precisamente, la libertad religiosa (Lee Miller, 2003, p. 3). Un valor que contrastaba con las persecuciones religiosas que, durante el gobierno de Jacobo I, sufrieron los puritanos (Coffey, 2013, pp. 112113). Ante la política represiva del rey británico, muchos buscaron refugio en Ámsterdam y en Leyden (Morton, [1669] (1855), p. 12). Sin embargo, la excesiva tolerancia que encontraron en los Países Bajos, reforzaría la idea de emigrar y emprender un largo viaje en pos de la anhelada Tierra de Promisión ${ }^{3}$. De esta manera, al cabo de algunos años de madurar la empresa, alrededor de ciento cincuenta puritanos se embarcaron en el legendario Mayflower, dejando el Viejo Mundo y creyéndose predestinados para ser los "Padres Peregrinos" de un mundo nuevo (Boettner, [1932] 2017, p. 160).

Cuando el 6 septiembre de 1620 el Mayflower zarpó del puerto de Plymouth, Inglaterra, a bordo de la nave viajaba también la cepa de un protestantismo de corte calvinista que, dos meses después, al término del viaje, se instalaría prontamente en la colonia de New Plymouth, en el

3 Sobre el ambiente religioso a inicios del siglo XVII en los Países Bajos, véase Armstrong y Chmielewski (2013, p. 126). Acerca de la consideración de la empresa colonizadora como un designio divino, véase Hirschfeld (2005, p. 40). 
sudeste del actual Estado de Massachusetts (Walsh, 1971, p. 32). Los líderes de la tripulación eran puritanos, pero de la secta brownista, como se indicará a continuación. Aunque, antes, parece conveniente hacer una precisión historiográfica:

La palabra "puritano" se asocia comúnmente a la "mojigatería”, "represión sexual", "prohibicionismo", "soplonería” (...). La imagen de los puritanos como teócratas, regicidas, quemadores de brujas, mata-indios y fanáticos perseguidores de herejías ha estado por mucho tiempo fijada en la cultura popular. Muchas de estas son imágenes distorsionadas — cuando no falsedades absolutas-; sin embargo, dichos estereotipos han quedado fuertemente arraigados. (Bremer, 2009, p. 1)

La crítica histórica, en cambio, ha sido unánime al considerar que los puritanos, a pesar de ser una minoría, constituyeron un grupo religioso influyente dentro del territorio británico (Brown, 2016, p. 622). Ellos, como miembros de la Iglesia de Inglaterra, reclamaban una reforma más enérgica por parte del Estado, de modo que se despojase a la liturgia, estructura, gobierno y constituciones eclesiales de la influencia del catolicismo romano ${ }^{4}$. Al constatar la inviabilidad de la pretendida reforma, muchos se agruparon para constituirse como un grupo religioso autónomo.

En los dos volúmenes que constituyen las Magnalia Christi Americana, del cronista puritano Cotton Mather (1663-1728), la empresa

4 Por ejemplo, la penalización a quienes no asistiesen a los oficios litúrgicos protestantes o que incumpliesen algunas normas eclesiales, tales como el ayuno y la abstinencia (Keniston McIntosh, 2012, p. 238). 
trasatlántica es enmarcada precisamente en un repertorio de elementos bíblicos: el viaje hacia América del Norte era visto como la reedición del éxodo del nuevo Israel. A través de sus páginas, Mather intenta reconstruir historiográficamente los inicios de Nueva Inglaterra para hacer notar a sus lectores "que no eran hipócritas, ni fanáticos entusiastas, ni meras víctimas de las sátiras, sino verdaderos protagonistas del drama de la redención" (Woolsey, 2003, p. 225) ${ }^{5}$. De esta manera, la teología que profesaban los Padres Peregrinos se convertiría en una de las características predominantes de la idiosincrasia estadounidense durante su período colonial ${ }^{6}$ :

El cultivo de un ethos público responsable fue una de las prioridades de los Puritanos (...). A pesar de que los clérigos tenían prohibido desempeñar un oficio en la administración civil, fue típico el verlos trabajar cercanamente a los magistrados en su empeño por modelar una sociedad que debía ser formalmente secular, pero profundamente cristiana. Instrumentos claves para conseguir este objetivo fueron no solamente las iglesias —a las que los ciudadanos estaban obligados a asistir-, sino también las escuelas que se constituyeron en casi todas las ciudades. (Bratt, 2009, p. 51)

5 Respecto a este tema, a través de las historiografías revisionistas de las últimas décadas, el puritanismo ha ido perdiendo aquel carácter radical que solía formar parte de su sello distintivo. Tan es así que para referirse a la época fundacional de los Estados Unidos se tiende a utilizar un "paradigma post-puritano", en favor del pluralismo religioso (Cohen, 1997, pp. 701-705).

6 La teología de los padres peregrinos ha sido considerada mayormente alineada con el calvinismo de los Hugonotes, aunque moderada (McGraw, 2011, p. 287). Al respecto, véase también Bunker (2011, p. 24). 
IN GOD WE TRUST: SOBRE LAS RAÍCES CRISTIANAS DEL CONCEPTO DE LIBERTAD RELIGIOSA EN LOS ESTADOS UNIDOS DE AMÉRICA

Lo que realmente marcaba distancia entre el pensamiento brownista de los Padres Peregrinos y el de otros protestantes calvinistas no se reflejaba tanto en materia doctrinal o moral, sino en el campo político. En efecto, aquellos proponían la separación de poderes, ante litteram, entre el Estado y las congregaciones eclesiásticas. Defendían el mismo principio que, más tarde, John Locke desarrollaría sistemáticamente en torno a la tolerancia religiosa. Junto con el valor de una democracia germinal, dicho respeto por las creencias religiosas personales sería uno de los fundamentos de los tripulantes del Mayflower. De hecho, antes de desembarcar en el Nuevo Mundo, los Peregrinos colonizadores firmaron un acta - a manera de contrato social - mediante la cual se comprometieron a seguir las reglas que se establecieran en lo sucesivo para el bien común, salvaguardando así el orden dentro del grupo y, en buena cuenta, su sobrevivencia.

\section{Del "Pacto del Mayflower" a la Constitución de los Estados Unidos} de América

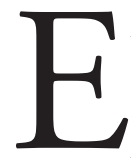

1 "Pacto del Mayflower" que fuera suscrito por los cuarentaiún tripulantes con derecho a voz y voto ${ }^{7}$, ha sido considerado como el primer documento en el que se deja constancia de la voluntad de un grupo humano por adoptar criterios democráticos y de tolerancia religiosa en tierras estadounidenses (Kemp, 2010, p. 3). Sin embargo, se ha señalado también que, a lo largo de la historia estadounidense, lo que en realidad se constata es una fluctuante muestra de tolerancia e intolerancia religiosa (Grenda, 2011, pp. 23-24). Dentro de quienes comparten este último enfoque, no faltan autores que señalan que se trataría de la construcción de un mito fundacional:

$7 \quad$ Vale decir, se excluía a las mujeres, niños y sirvientes (Arnoux Haxtun, 2004, pp. 5-8). 
El mito de la fundación religiosa de [los Estados Unidos de] América emergió a inicios del siglo XIX, en la búsqueda de una identidad nacional; una identidad que necesitaba validarse con la bendición de Dios (...). Las primeras manifestaciones del mito se dieron a través de las segunda y tercera generaciones de los Puritanos apologistas, quienes se esforzaron por entender y justificar la importancia de la misión puritana. (Green, 2015, p. 243)

Independientemente de su influencia ulterior, los colonos de New Plymouth constituyeron el primer grupo que pudo asentarse con éxito en Nueva Inglaterra. Asimismo, el Pacto del Mayflower "estableció en dicho asentamiento un gobierno democrático a través del mutuo consentimiento de los hombres libres de la colonia" (Dupertuis Bangs, 2008, p. 61). Si bien es cierto en el citado documento no se hace referencia a alguna religión ฐ específica, el contenido es explícito a la hora de citar a Dios como principio y fin de la empresa colonizadora:

En el nombre de Dios. Amén. Nosotros, los suscritos, súbditos leales de nuestro soberano señor Jacobo, por la gracia de Dios, rey de Gran Bretaña, Francia e Irlanda, defensor de la fe, etc., habiendo emprendido un viaje - para la gloria de Dios y el avance de la fe cristiana, y para honrar a nuestro rey y a nuestra nación - para establecer la primera colonia en las zonas nortes de Virginia, por medio de la presente, solemnemente y de mutuo acuerdo, ante la presencia de Dios y de nosotros mismos, nos unimos en alianza para formar un cuerpo político civil, para 
alcanzar un mejor orden y para nuestra preservación y para el cumplimiento de los fines antes mencionados. (Bowman, 1920, p. 6)

El hecho de no explicitar la denominación cristiana de pertenencia es una ausencia no secundaria; más aún, es un silencio de redacción del todo elocuente $^{8}$. Cuestiona, en primer lugar, la supuesta condición radicalizada del puritanismo que profesaba mayoritariamente la tripulación del Mayflower; y deja entrever un carácter conciliador por parte de los firmantes. Por otra parte, pone de manifiesto el espíritu abiertamente religioso del cual se deseaba dejar constancia: pese a la brevedad del documento, se menciona a Dios cuatro veces. Además, respetuosos de las leyes, se presentaron como súbditos del rey Jacobo I, de quien, como se ha dicho, habían sufrido persecución por motivos religiosos (Cragg, 2011, pp. 51-52).

¿Cuánto hay de mito y cuánto corresponde a lo que realmente ocurrió? Al historiador le compete (dis)cernir, en la medida de lo posible, tomando en cuenta los indicios más sólidos, aquellos que podrían ajustarse mejor a la mentalidad y a los cánones de comportamiento más verosímiles de aquel tiempo?. No se ha pretendido pronunciar un juicio categórico sobre los eventos fundacionales de los Estados Unidos de América. Abunda una bibliografía jaloneada entre historiografías contrapuestas, que deben ser

8 Sobre la importancia de los silencios documentarios para el historiador, véase De Certeau (1975, p. 67).

9 El anacronismo es el adversario que más veces se ha enfrentado a las ciencias históricas sociales, aunque no el más feroz. Este puesto lo ocupa aquella apología que manipula las fuentes históricas para refrendar sus propias elaboraciones mentales que, a su vez, obedecen a intereses ideológicos particulares (Rancière, 1992, p. 66). 
ponderadas por un análisis crítico de los factores políticos, ideológicos y económicos que entraron en juego ${ }^{10}$.

En cualquier escenario, el "factor religioso" ha tenido un claro protagonismo en la forja de la historia de los Estados Unidos de América (Furseth \& Repstad, 2006, p. 103). Dios ha sido el destinatario principal de las gestas de los ciudadanos estadounidenses: a Él se le han tributado los mayores éxitos de la nación ${ }^{11}$; su Nombre ha sido citado en los discursos más remarcables ${ }^{12}$; una lapidaria declaración de fe (In God we Trust) ha sido acuñada en su moneda corriente ${ }^{13}$. Sin embargo, el Nombre de aquel mismo Dios ha sido también mentado para manipular a la opinión pública, pretendiendo justificar los crímenes más execrables, desde el genocidio en los albores de su empresa colonizadora hasta el fratricidio en la más reciente historia contemporánea ${ }^{14}$. En todo caso, no obstante el uso y abuso del nombre divino, Dios no ha dejado de ser el inspirador de los valores más altos de la nación estadounidense ${ }^{15}$ :

10 Sobre la contraposición historiográfica de la tripulación del Mayflower, véase Bailyn (2017, p. 323). Un análisis crítico de su contexto histórico puede encontrarse en Martin (1989, p. 191).

11 Como la odisea al espacio (Hansen, 2006, p. 629).

12 Por ejemplo, el de Martin Luther King (Miller, 2012, p. 155).

13 Para una breve explicación del origen de la elección del término, véase Davison (2006, pp. 1066-1067).

14 Sobre la manipulación del nombre de Dios para justificar los métodos colonizadores empleados en la conquista de Norte América, véase Lindsay (2012, p. 65) y Pruin, Scheider y Zachhuber (2007, p. 14). Para una aplicación en clave contemporánea, puede verse en Block (2012, p. 8).

15 La democracia, la igualdad y la justicia son valores que, fundamentados sobre la base de la libertad, constituyen "la esencia de la filosofía política contemporánea" (Matravers y Meyer, 2011, p. 1). Sobre el valor de la libertad en los Estados Unidos, véase Nelson (2005, p. 1). 
Las encuestas han mostrado permanentemente que la religión ha permanecido como el principal valor en la vida de los estadounidenses. En una encuesta llevada a cabo por Pew Research Center for the People and the Press, el 64\% de los encuestados afirmaron que la religión es muy importante en sus vidas (...). El estudio también indicaba que el $90 \%$ de los estadounidenses ora al menos una vez por semana - con un 59\% en el grupo de los que reza todos los días e incluso varias veces al día-; el 60\% asiste a un servicio religioso por lo menos una vez al mes — con un $43 \%$ en el grupo de los que asiste a un servicio religioso cada semana. (Murray, 2008, p. 3)

La historia de la propia Constitución de los Estados Unidos de América es un elemento clave para la comprensión del valor histórico que la libertad religiosa ha tenido en dicho país. En efecto, desde que esta entrara en vigor en 1789, la Constitución ha tenido 27 intervenciones enmendadoras (Vile, 2003, p. XXII). Una de las más significativas ha sido la Primera Enmienda, mediante la cual se logró disipar cualquier duda con respecto a la libertad y a la tolerancia religiosa en la sociedad estadounidense. A través de ella se estableció la libertad religiosa como un derecho fundamental que debía ser protegido por el Estado: El Congreso fue impedido de legislar a favor o en contra del establecimiento de una religión o de su libre ejercicio ${ }^{16}$. De esta manera, imponiendo a la autoridad gubernamental una serie de límites, la Primera Enmienda se constituyó en un marco legislativo clave para propiciar una vitalidad continua de la religiosidad norteamericana, en medio de su amplia diversidad ${ }^{17}$.

16 Sobre el proceso evolutivo en la redacción de la Primera Enmienda, véase Haynes, Chaltain y Glisson (2006, pp. 47-48).

17 Sobre el sentido de la primera enmienda, véase Abrams (2017, p. 11). Acerca del 


\section{La Libertad Religiosa en clave contemporánea}

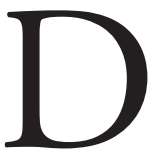

os figuras contemporáneas podrían encarnar la lucha por la salvaguardia de la libertad religiosa. Ambos, coincidentemente, han sido considerados expertos en el arte de la comunicación: Ronald Reagan y Jorge Mario Bergoglio. El primero, presidente de los Estados Unidos desde 1981 hasta 1989 y fallecido en el 2004, afirmó lo siguiente:

El Pacto del Mayflower empezó con estas palabras: "En el nombre de Dios...”. La Declaración de la Independencia apeló a la "Naturaleza de Dios", al "Creador" y al "Supremo Juez del universo" (...). James Madison, en los Ensayos Federalistas, admitió que en la creación de nuestra República percibió la mano del Altísimo. John Jay, el primer Jefe de la Suprema Corte de Justicia, advirtió que nunca debíamos a Dios, de quien recibimos sus bendiciones. George Washington se refirió al lugar profundo e irreemplazable que ocupa la religión en el corazón de la nación (...); siete años antes, Francia había instaurado un gobierno puramente secular. Este gobierno se fundaría en la razón antes que en la ley de Dios. Hacia 1796 la Revolución Francesa conocería el Gobierno del Terror (...). Creo que George Washington llegó a entender que la Ciudad del Hombre no podría sobrevivir sin la Ciudad de Dios, que la Ciudad Visible perecería sin la Ciudad Invisible. (Reagan, 1986, p. 1166) ${ }^{18}$

potencial carácter conflictual de la religión en los Estados Unidos, véase Putnam y Campbell (2010, p. 519).

18 El discurso del expresidente norteamericano fue recordado por el senador Robert B. Aderholt en sus honras fúnebres (Joint Committee on Printing Congress, 2005, pp. 113-114). 
Por su parte, el papa Francisco, en su discurso al Congreso de los Estados Unidos de América en septiembre del 2015, resaltó reiteradamente la importancia del derecho de la persona a la libertad. En el marco de un memorable e intenso viaje apostólico, en el que también visitó Cuba y dirigió al mundo un mensaje profético desde la Sede de la Organización de las Naciones Unidas, sus palabras resonaron con fuerza en el hemiciclo de Washington D.C. ${ }^{19}$. Para el papa latinoamericano, la grandeza de una nación reside en su capacidad para defender el valor de las libertades personales de quienes la componen:

Somos conscientes de que ninguna religión es inmune a diversas formas de aberración individual o de extremismo ideológico. Esto nos urge a estar atentos frente a cualquier tipo de fundamentalismo de índole religiosa o del tipo que fuere. Combatir la violencia perpetrada bajo el nombre de una religión, una ideología, o un sistema económico y, al mismo tiempo, proteger la libertad de las religiones, de las ideas, de las personas requiere un delicado equilibrio en el que tenemos que trabajar. (Francisco, 2015)

\section{A modo de Conclusión}

T $\begin{aligned} & \text { emos visto cómo el protestantismo de corte calvinista, de la secta } \\ & \text { brownista, se instaló en New Plymouth (sudeste del actual estado } \\ & \text { de Massachusetts), lugar desde el que se propagaría una teología }\end{aligned}$

19 Véase un análisis de dicho discurso en Piccone Camere (2018, pp. 327-350). 
política sobre la cual se cimentaría la idiosincrasia estadounidense durante su período colonial, base de la cohesión social hodierna. Uno de los puntos en los que se puso mayor énfasis fue el referido a la tolerancia religiosa. Este principio quedó manifestado de una manera implícita en el llamado "Pacto de Mayflower" y se hizo explícito a través de la primera enmienda de la Constitución estadounidense. Si bien la presentación histórica de los hechos ha sido objeto de múltiples interpretaciones, existe consenso para afirmar que el "factor religioso" tuvo y sigue teniendo un claro protagonismo en la forja de la historia de los Estados Unidos de América. Esto último ha sido un potente catalizador de empresas heroicas, pero también se ha prestado para justificar actos incoherentes con la fe profesada.

\section{REFERENCIAS}

Abrams, F. (2017). The Soul of the First Amendment. New Haven, Estados Unidos: Yale University Press.

Armstrong, C., \& Chmielewski, L. (2013). The Atlantic Experience: Peoples, Places, Ideas. New York, Estados Unidos: Palgrave Macmillan.

Armstrong, N., \& Tennenhouse, L. (1992). The Imaginary Puritan: Literature, Intellectual Labor, and the Origins of Personal Life. Berkeley, Estados Unidos: University of California Press.

Arnoux Haxtun, A. (2004). Signers of the Mayflower Compact. Baltimore, Estados Unidos: Genealogical Publishing Company.

Bailyn, B. (2017). The Ideological Origins of the American Revolution. Cambridge, Estados Unidos: Harvard University Press. 
IN GOD WE TRUST: SOBRE LAS RAÍCES CRISTIANAS DEL CONCEPTO DE LIBERTAD RELIGIOSA EN LOS ESTADOS UNIDOS DE AMÉRICA

Block, T. (2012). A Fatal Addiction: War in the Name of God. New York, Estados Unidos: Algora Publishing.

Boettner, L. [Phillipsburg, 1932] (2017). The Reformed Doctrine of Predestination (Ed. A. Uyl). Woodstock, Canadá: Devoted Publishing.

Bowman, G. (Ed.) (1920). The Mayflower compact and its signers: with facsimiles and a list of the Mayflower passengers. Boston, Estados Unidos: Massachusetts Society of Mayflower Descendants.

Bratt, J. (2009). Calvinism in North America. En M. Hirzel \& M. Sallmann (Eds.), John Calvin's Impact on Church and Society, 1509-2009 (pp. 49-66). Grand Rapids, Estados Unidos: Eerdmans.

Bremer, F. (2009). Puritanism: A Very Short Introduction. New York, Estados Unidos: Oxford University Press.

Brown, T. (2016). Anglican Way or Ways? En M. Chapman, S. Clarke \& M. Percy (Eds.), The Oxford Handbook of Anglican Studies (pp. 620635). New York, Estados Unidos: Oxford University Press.

Bunker, N. (2011). Making Haste from Babylon: The Mayflower Pilgrims and their World: A New History. London, Reino Unido: Pimlico.

Coffey, J. (2013). Persecution and Toleration in Protestant England 15581689. New York, Estados Unidos: Routledge.

Cohen, C. (1997). The Post-Puritan paradigm in Early American Religious History. William and Mary Quarterly, 54, 701-705.

Cragg, G. (2011). Puritanism in the Period of the Great Persecution 16601688. New York, Estados Unidos: Cambridge University Press. 
Davison, D. (2006). National Motto "In God We Trust". En P. Finkelman (Ed.), Encyclopedia of American Civil Liberties. Vol. I: $A-F$ (pp. 1066-1067). New York, Estados Unidos: Routledge.

De Certeau, M. (1975). L'écriture de l'histoire. París, Francia: Gallimard.

Dupertuis Bangs, J. (2008). Re-Bunking the Pilgrims. En D. Yerxa (Ed.), Recent Themes in Early American History: Historians in Conversation (pp. 59-66). Columbia, Estados Unidos: The University of South Carolina Press.

Forster, G. (2005). John Locke's Politics of Moral Consensus. New York, Estados Unidos: Cambridge University Press.

Francisco. (2015). Discurso del Santo Padre en su Visita al Congreso de los Estados Unidos de América (Washington D.C., 24 de septiembre del 2015). La Santa Sede. Recuperado de http://w2.vatican.va/ content/francesco/es/speeches/2015/september/documents/papafrancesco_20150924_usa-us-congress.html

Furseth, I., \& Repstad, P. (2006). An Introduction to the Sociology of Religion: Classical and Contemporary. Hants, Reino Unido: Ashgate.

Glaser, E. (Ed.) (2014). Religious Tolerance in the Atlantic World: Early Modern and Contemporary Perspectives. New York, Estados Unidos: Palgrave-Macmillan.

Green, S. (2015). Inventing a Christian America: The Myth of the Religious Founding. New York, Estados Unidos: Oxford University Press.

Grenda, C. (2011). Faith, Reason and Enlightment. The Cultural Sources of Toleration in Early America. En C. Beneke \& C. Grenda (Eds.), The First Prejudice: Religious Tolerance and Intolerance in Early 
IN GOD WE TRUST: SOBRE LAS RAÍCES CRISTIANAS DEL CONCEPTO DE LIBERTAD RELIGIOSA EN LOS ESTADOS UNIDOS DE AMÉRICA

America (pp. 23-52). Philadelphia, Estados Unidos: University of Pennsylvania Press.

Hansen, J. (2006). First Man: The Life of Neil A. Armstrong. New York, Estados Unidos: Simon \& Schuster Paperbacks.

Haynes, C., Chaltain, S., \& Glisson, S. (2006). First freedoms: a documentary history of First Amendment Rights in America. New York, Estados Unidos: Oxford University Press.

Hirschfeld, F. (2005). George Washington and the Jews. Newark, Estados Unidos: University of Delaware Press.

Joint Committee on Printing Congress. (2005). Memorial Services in the Congress of the United States and Tributes in eulogy of Ronald Reagan, late a President of the United States. Washington D.C., Estados Unidos: United States Government Printing Office.

Kemp, R. (2010). Documents of American Democracy: A Collection of Essential Works. Jefferson, Estados Unidos: McFarland \& Company.

Keniston McIntosh, M. (2012). Poor Relief in England, 1350-1600. New York, Estados Unidos: Cambridge University Press.

Lee Miller, W. (2003). The First Liberty: America's Foundation in Religious Freedom, Expanded and Updated. Washington DC, Estados Unidos: Georgetown University Press.

Lindsay, B. (2012). Murder State: California's Native American Genocide, 1846-1873. Lincoln, Estados Unidos: University of Nebraska Press. Locke, J. [Londres, 1689] (2013). A Letter Concerning Toleration. K. Walters (Ed.). Toronto, Canadá: Broadview Editions.

Marshall, J. (2006). John Locke, Toleration and Early Enlightenment Culture. New York, Estados Unidos: Cambridge University Press. 
Martin, J.-P. (1989). Le puritanisme américain en Nouvelle-Angleterre: 16201693. Talence, Francia: Presses Universitaires de Bordeaux.

Matravers, M., \& Meyer, L. (2011). Introduction. En M. Matravers \& L. Meyer (Eds.), Democracy, Equality, and Justice (pp. 1-16). New York, Estados Unidos: Routledge.

McGraw, P. (2011). The memory of the Huguenots in North America: Protestant history and polemic. En D. Trim (Ed.), The Huguenots: History and memory in transnational context: Essays in honour and memory of Walter C. Utt (pp. 285-304). Leiden, Países Bajos: Brill.

Miller, K. (2012). Martin Luther King's Biblical Epic: His Final, Great Speech. New York, Estados Unidos: University Press of Mississippi.

Morton, N. [Cambridge, 1669] (1855). New England's Memorial. Or, a Brief Relation of the Most Memorable and Remarkable Passages of the Providence of God, Manifested to the Planters of New-England in America: With Special Reference to the First Colony Thereof, called New-Plimouth. Boston, Estados Unidos: Congregational Board of Publication.

Murray, B. (2008). Religious Liberty in America: The First Amendment in Historical and Contemporary Perspective. Amherst, Estados Unidos: University of Massachusetts Press.

Nelson, S. (2005). Beyond the First Amendment: The Politics of Free Speech and Pluralism. Baltimore, Estados Unidos: The John Hopkins University Press.

Piccone Camere, C. (2018). "Les hablo como hijo de inmigrantes": Los ecos históricos del discurso del Papa Francisco ante el Congreso de 
IN GOD WE TRUST: SOBRE LAS RAÍCES CRISTIANAS DEL CONCEPTO DE LIBERTAD RELIGIOSA EN LOS ESTADOS UNIDOS DE AMÉRICA

los Estados Unidos (24 de septiembre de 2015). Revista Teológica Limense, 3, 327-350.

Pruin, D., Schieder, R., \& Zachhuber, J. (2007). Introduction. En D. Pruin, R. Schieder \& J. Zachhuber (Eds.), Religion and Politics in the United States and Germany (pp. 7-16). Berlín, Alemania: Lit verlag.

Putnam, R., \& Campbell, D. (2010). American Grace: How Religion Divides and Unites Us. New York, Estados Unidos: Simon \& Schuster Paperbacks.

Rancière, J. (1992). Les Noms de l'histoire: essai de poétique du savoir. París, Francia: Éditions du Seuil.

Reagan, R. (1986). Remarks at an Ecumenical Prayer Breakfast in Dallas, Texas (August 23, 1984). En Public Papers of the Presidents of the United States: Ronald Reagan, 1984 (pp. 1165-1168). Washington D.C., Estados Unidos: Federal Register Division, National Archives and Records Service, General Services Administration.

Vile, J. (2003). Encyclopedia of Constitutional Amendments, Proposed Amendments, and Amending Issues, 1789-2002. Santa Barbara, Estados Unidos: ABC-CLIO.

Walsh, J. (1971). The Mayflower compact, November 11, 1620: The first democratic document in America. New York, Estados Unidos: F. Watts.

Woolsey, S. (2003). Staging a Puritan Saint: Cotton Mather's Magnalia Christi Americana. En L. Lunger Knoppers (Ed.), Puritanism and Its Discontents (pp. 210-230). Newark, Estados Unidos: University of Delaware. 
